\title{
Percutaneous Transvenous Stent Implantation to External Iliac Vein Stenosis in a Patient with Retroperitoneal Fibrosis
}

\author{
Hidenobu Okuyama ${ }^{1}$, Osamu Hirono ${ }^{1}$, Daisuke Ishigaki ${ }^{1}$, Koichi Yuki ${ }^{1}$ and Isao Kubota ${ }^{2}$
}

\begin{abstract}
A 59-year-old man visited our hospital due to right leg edema and right leg pain. Computed tomography revealed that the circumferential enhancement of bilateral external iliac arteries by soft tissue that had similar density as the adjacent psoas muscle and that the right external iliac vein that was constricted by those tissues. The patient was diagnosed as retroperitoneal fibrosis. He underwent stent implantation to the right external iliac vein stenosis and steroid therapy. His right leg edema and pain was immediately improved after the stent implantation and he achieved remission.
\end{abstract}

Key words: retroperitoneal fibrosis, external iliac vein stenosis, stent implantation, steroid therapy

(Inter Med 49: 913-918, 2010)

(DOI: 10.2169/internalmedicine.49.3185)

\section{Introduction}

It is well known that retroperitoneal fibrosis (RPF) is a rare disease with a low incidence rate $(1 / 1,000,000$ people). Since 1948, classical idiopathic RPF has been thought to be a urological disease that is characterized by fibrous tissue and inflammatory cells invasion to the ureter, abdominal aorta and vena cava and urinary tract obstruction $(1,2)$. Although surgical ureterolysis and oral administration of steroids is used in these patients, the treatment strategy for venous stenosis and the effective dose of steroids have not yet been clearly shown. Here, we describe a patient diagnosed as RPF in whom the stent implantation to external iliac vein stenosis and oral administration of steroid were effective.

\section{Case Report}

A 59-year-old man was admitted with severe leg edema and pain in his right leg in July 2008. He had been treated with oral administration of beta-blocker (atenolol $50 \mathrm{mg}$ ) for 3 years due for hypertension. He had no specific family history.

He was $170 \mathrm{~cm}$ tall with a body weight of $80 \mathrm{~kg}$. His blood pressure was $132 / 70 \mathrm{mmHg}$ and pulse rate was 62 / min. He had no fever, anemia or jaundice. No pathological findings were observed in the chest. A high-pitched vascular murmur was auscultated from the umbilical region to bilateral groin. He had right leg pitting edema with superficial vessel dilatation. Laboratory investigation gave the following results: white blood cell count $7,290 / \mu \mathrm{L}$, the red blood cell count $4.82 \times 10^{6} / \mu \mathrm{L}$, hemoglobin $12.2 \mathrm{~g} / \mathrm{dL}$, hematocrit $37.9 \%$, platelet count $15.1 \times 10^{4} / \mu \mathrm{L}$, aspartate aminotransferase $25 \mathrm{IU} / \mathrm{L}$ (normal<33), alanine aminotransferase $22 \mathrm{IU} /$ L (normal<30), $\gamma$-glutamyltranspeptidase 42 IU/L (normal< 71), alkaline phosphatase 225 IU/L (normal <147), lactate dehydrogenase $163 \mathrm{IU} / \mathrm{L}$ (normal<425), blood urea nitrogen $14 \mathrm{mg} / \mathrm{dL}$ (normal<20), creatinine $0.8 \mathrm{mg} / \mathrm{dL}$ (normal<1.0). There were no findings of inflammation: erythrocyte sedimentation rate $4 \mathrm{~mm} /$ hour (normal $<10$ ), C-reactive protein $0.16 \mathrm{mg} / \mathrm{dL}$ (normal <1.0). On urinalysis, urinary protein was not detected. On thyroid function tests, the levels of thyrotropin (TSH), free triiodothyronine (fT3) and free thyroxine (fT4) were within normal range: TSH $1.2 \mu \mathrm{U} / \mathrm{mL}$ (normal 0.4-5.0), fT3 $3.2 \mathrm{ng} / \mathrm{mL}$ (normal 2.4-4.3), fT4 1.1 $\mathrm{ng} / \mathrm{dL}$ (normal 0.8-1.8). The levels of tumor markers were within normal range: cytokeratin 19 fragment $0.85 \mathrm{ng} / \mathrm{mL}$ (normal<2.2), carcinoembryonic antigen $1.2 \mathrm{ng} / \mathrm{mL}$ (normal $<3$ ), carbohydrate antigen $19-95.9 \mathrm{U} / \mathrm{mL}($ normal<24), soluble interleukin-2 receptor $383 \mathrm{U} / \mathrm{mL}$ (normal<650). The

${ }^{1}$ Division of Cardiology, Yamagata Prefectural Shinjyo Hospital, Shinjo and ${ }^{2}$ Department of Cardiology, Pulmonology and Nephrology, Yamagata University School of Medicine, Yamagata

Received for publication November 25, 2009; Accepted for publication January 21, 2010

Correspondence to Dr. Hidenobu Okuyama, hiokuyama-circ@umin.net 


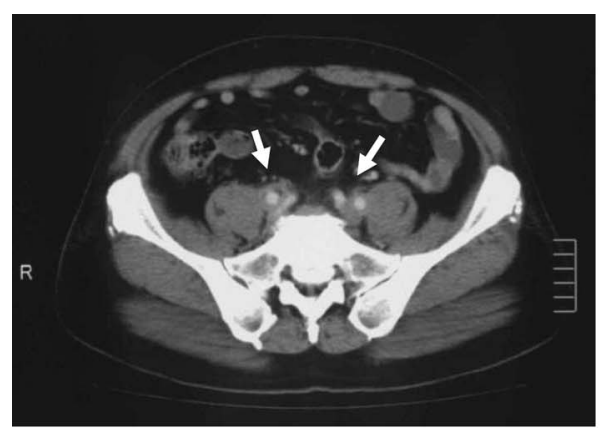

A

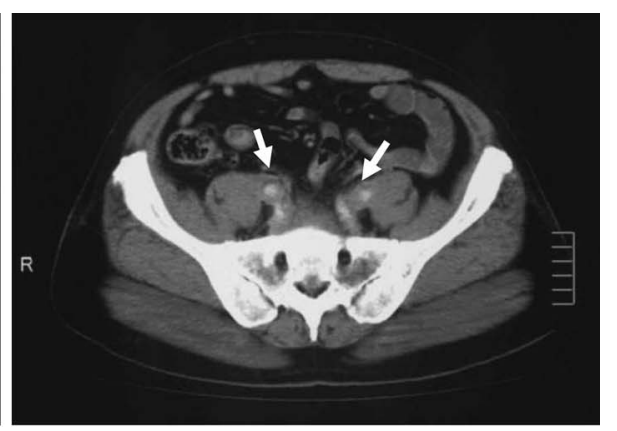

B

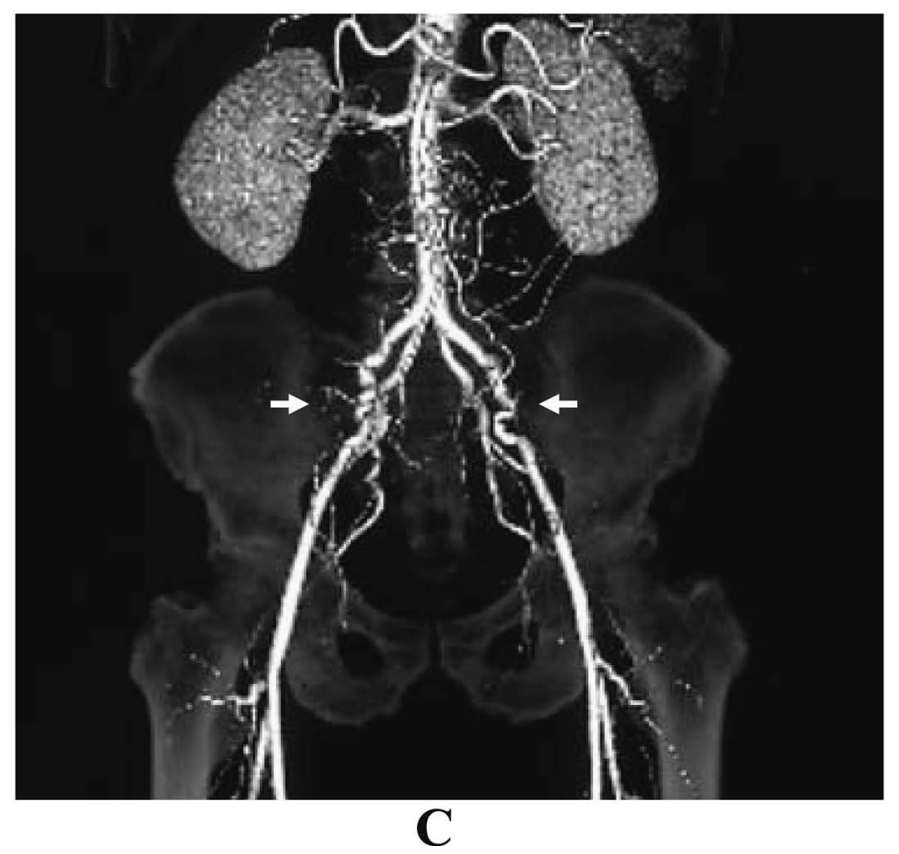

Figure 1. Abdominal contrast-enhanced computed tomography (CT) revealed circumferential enhancement of bilateral external iliac arteries by soft tissue (arrow) of a similar density to that of the adjacent psoas muscle and tha the right external iliac vein that was constricted by those tissues (A, B). The 3-directional CT angiography showed that bilateral external iliac artery was markedly serpentine (arrow) without calcification (C).

levels of serum complement, immunoglobulin and various autoantibodies were also within the normal range: complement $3119 \mathrm{mg} / \mathrm{dL}$ (normal<140), complement $424 \mathrm{mg} / \mathrm{dL}$ (normal<40), homolytic complement activity $38 \mathrm{U} / \mathrm{mL}$ (normal<45), immunoglobulin G 1,310 mg/dL (normal<1,349), immunoglobulin A 308 mg/dL (normal<312), immunoglobulin M $62 \mathrm{mg} / \mathrm{dL}$ (normal <352), antinuclear antibody <40fold (normal<40-fold), proteinase-3 anti-neutrophil cytoplasmic antibody $<10 \mathrm{EU}$ (normal $<10 \mathrm{EU}$ ), myeloperoxidase anti-neutrophil cytoplasmic antibody $<10$ EU (normal $<10$ EU). The levels of coagulation markers were also within the normal range: fibrinogen $273 \mathrm{mg} / \mathrm{dL}$ (normal 200-400), fibrinogen degradation products $10.1 \mu \mathrm{g} / \mathrm{mL}$ (normal<11), Ddimer $0.4 \mu \mathrm{g} / \mathrm{mL}$ (normal <1.0), antithombin III 92\% (normal 80-120), РT\% 92\% (normal 70-120), АРTT\% 97\% (normal 70-130).

Chest X-ray showed no abnormal findings. Electrocardiogram showed sinus rhythm and no significant ST-T change. Ankle-Brachial Index and pulse wave velocity were within the normal range (right side: 1.12 , and $11.7 \mathrm{~m} / \mathrm{s}$; left side:
1.02 , and $10.2 \mathrm{~m} / \mathrm{s})$. Transthoracic echocardiography showed a normal ejection fraction of $63 \%$ and no signs of heart failure. Venous ultrasonography showed the right external iliac vein stenosis and the peak flow velocity at this site was 210 $\mathrm{cm} / \mathrm{s}$. Abdominal contrast-enhanced computed tomography (CT) revealed that the circumferential enhancement of bilateral external iliac artery by soft tissue that had a similar density as the adjacent psoas muscle and the right external iliac vein that was constricted by those tissues (Fig. 1). There were no significant pathological findings in the urinary tract, abdominal aorta and inferior vena cava. The 3directional CT angiography showed that bilateral external iliac arteries were markedly serpentine without calcification (Fig. 1). Magnetic resonance imaging (MRI) revealed high intensity signals at the right external iliac vein due to the disappearance of flow in T2-weighted imaging (Fig. 2A) and high intensity signals at the circumferential tissue of bilateral external iliac arteries on T1-weighted imaging and gadolinium enhanced MRI imaging (Fig. 2B and 2C).

We clinically diagnosed this patient as retroperitoneal fi- 


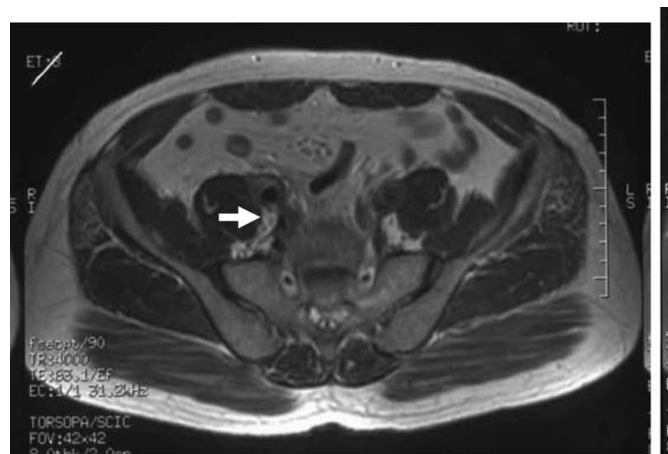

A

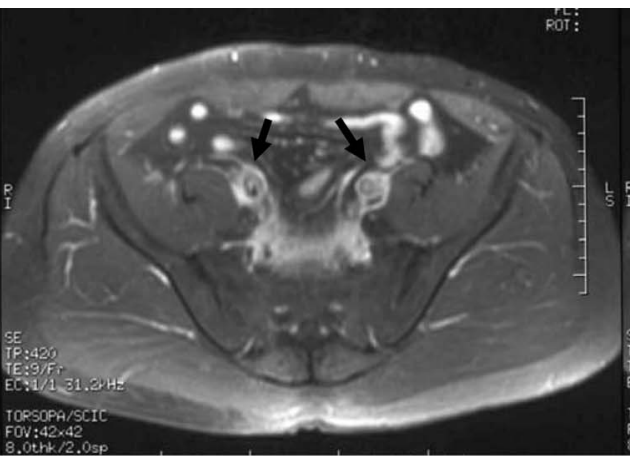

B

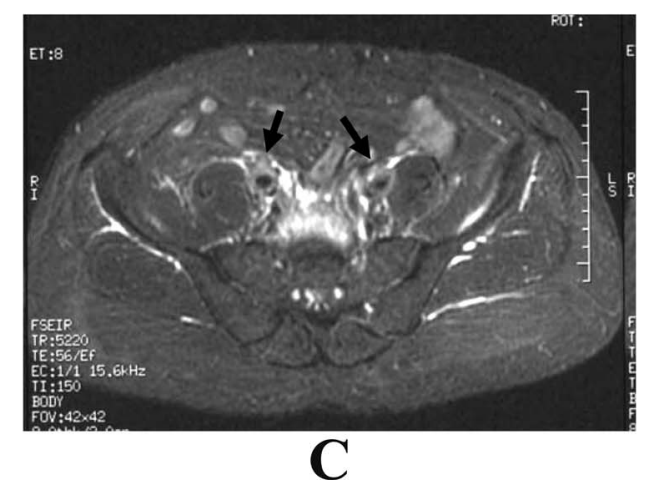

Figure 2. Magnetic resonance imaging (MRI) revealed high intensity signals (arrow) at the right external iliac vein due to disappearance of flow void on T2-weighted imaging (A) and high intensity signals at the circumferential tissue of bilateral external iliac artery on T1-weighted imaging (arrow) (B). Gadolinium enhanced MRI showed high intensity signals (arrow) at the circumferential tissue of bilateral external iliac arteries (C).

brosis with right external iliac vein stenosis. It has been reported that beta-blockers might be the cause of secondary RPF (3), therefore, atenorol was withdrawn from remedies of hypertension and oral administrations of enarapril $(5 \mathrm{mg} /$ day) and predonisolone $(30 \mathrm{mg} /$ day $)$ was started. He underwent right external iliac venography and stent implantation to the right external iliac vein in late July 2008. In venography, the mid-portion of the right external iliac vein had a severe stenosis of $8 \mathrm{~cm}$ in length and a filling delay of contrast media with no thrombus formation (Fig. 3A, 3B). Therefore, after the insertion of the vascular sheath, we injected heparin sodium at a dose of 5,000 unit intravenously and performed venous stent implantation to this site (SMART stent ${ }^{\mathrm{R}}: 9 \mathrm{~mm}$ in diameter and $40 \mathrm{~mm}$ in length, and $60 \mathrm{~mm}$ in length). We started oral warfarin therapy after the stent implantation and adjusted the dose of warfarin to achieve and maintain PT-INR in the range of 1.6 to 2.6 to keep long-term patency of the stent. After the stent implantation, blood flow of the right external iliac vein was markedly improved (Fig. 3C, 3D). His right leg edema and pain disappeared 2 days after the stent implantation.

He was treated with oral administration of predonisolone with a dose of $30 \mathrm{mg}$ for 4 weeks. Four weeks after the treatment with steroid, the patient underwent contrastenhanced CT and venous ultrasonography to evaluate the efficacy of steroid therapy. CT showed the regression of retroperitoneal fibrosis in responding well to steroid therapy (Fig. 4) and venous ultrasonography showed that the peak flow velocity at the external iliac vein was decreased to 20 $\mathrm{cm} / \mathrm{s}$ compared with $210 \mathrm{~cm} / \mathrm{s}$ on admission. We confirmed the efficacy of steroid therapy and reduced the dose of predonisolone by $5 \mathrm{mg}$ per 4 weeks. He was discharged from our hospital in September 2008. We performed enhanced CT at 3 and 12 months after the stent implantation and confirmed the patency of this vascular stent.

\section{Discussion}

According to the previous reports on pathological examinations of the RPF, this disease has been characterized by deteriorated retroperitoneal structure, which has chronic inflammation and fibroblast proliferation with excessive extracellular matrix, involves ureters, abdominal aorta, vena cava, and pancreas (most commonly ureters) $(1,2)$. It has been reported that about $90 \%$ of cases with RPF had lower back pain and/or abdominal pain as a chief complaint due to unilateral or bilateral urinary tract obstruction (4). As an uncommon case, invasion and compression to the iliac artery and/or vein by proliferated retroperitoneal tissue might cause intermittent claudication, leg edema and/or thrombosis (4). Koep and Zuidema reported that only $2.4 \%$ of their experienced 491 cases with RPF had complications with iliac artery and/or vein stenosis (5). In the present case, we experienced a rare case of RPF who had complications with right leg edema and severe pain due to right external iliac vein stenosis without iliac artery stenosis and urinary tract ob- 


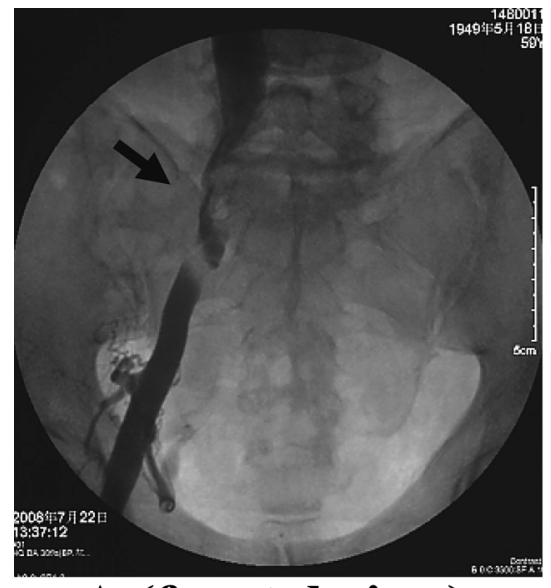

A (frontal view)

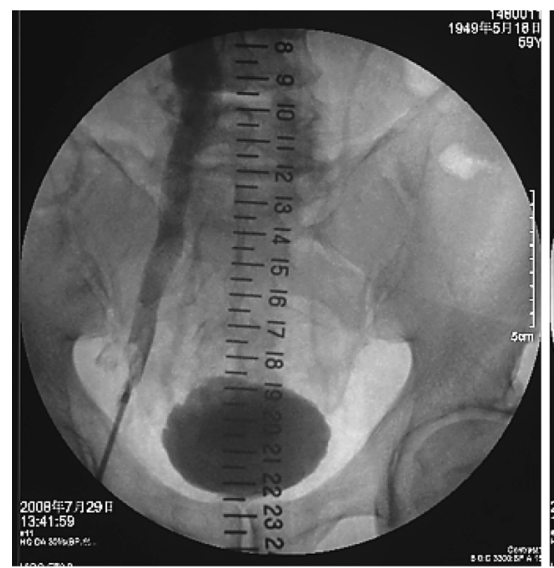

C (frontal view)

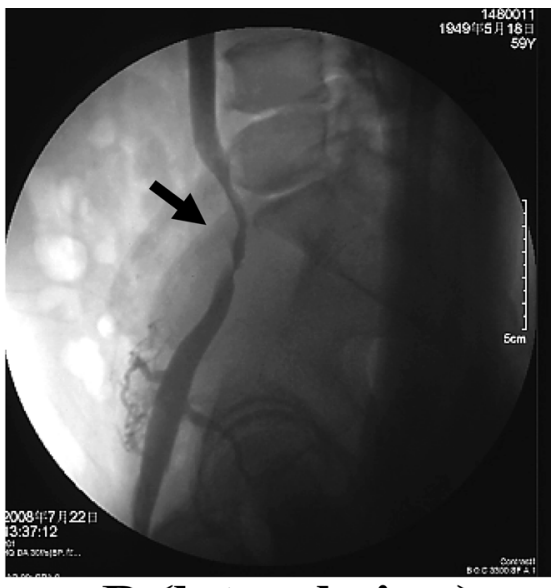

B (lateral view)

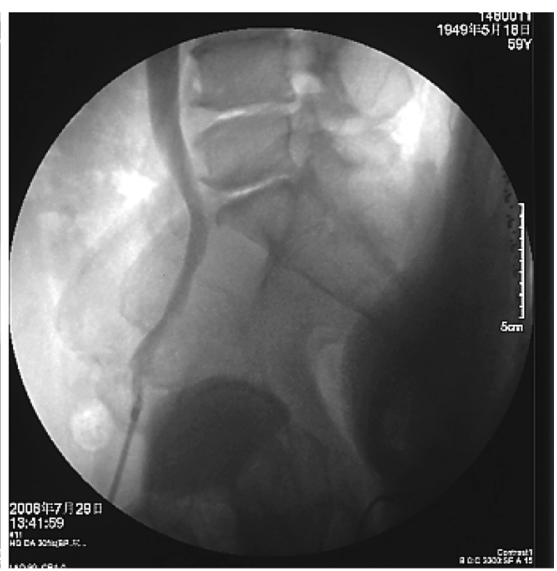

D (lateral view)

Figure 3. On venography, there was a severe stenosis of about $8 \mathrm{~cm}$ in length (arrow) and filing delay of contrast media in the mid-portion of the right external iliac vein (A, B). After the stent implantation, stenosis of the right external iliac vein was markedly improved $(C, D)$.

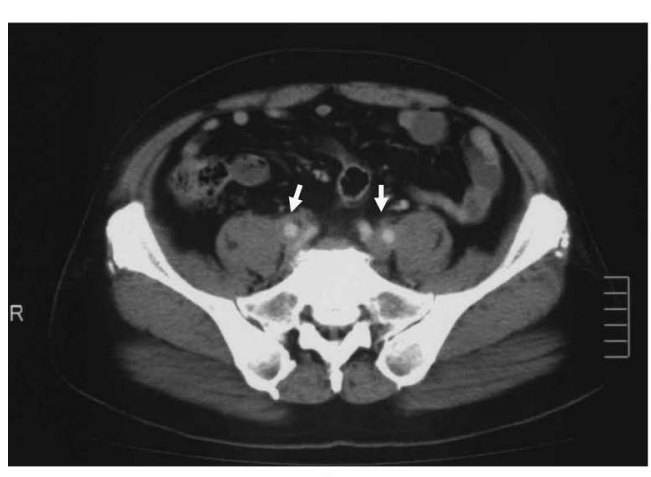

A

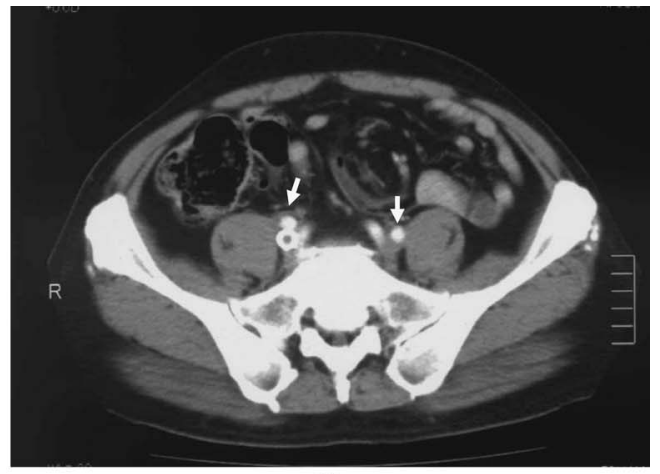

B

Figure 4. CT showed the regression of retroperitoneal fibrosis (arrow) in response to the steroid therapy (A: on admission, B: 4 weeks after the steroid therapy and stent implantation to right external iliac vein)

struction.

RPF was commonly diagnosed by ultrasonography, CT, MRI and intravenous urography. A retroperitoneal biopsy might be useful to confirm the diagnosis of RPF, however, Vaglio et al and Baker et al previously suggested that biopsy was not necessary if CT or MRI revealed typical findings of RPF $(3,6)$. Contrast-enhanced CT is a useful tool to evaluate the presence of hydronephrosis and lymphadenopathy, and invasion of proliferated retroperitoneal tissue to abdominal aorta and inferior vena cava. RPF has a similar density to as psoas muscle on CT $(7,8)$. MRI is commonly used in the differential diagnosis of this disease and malignancy (9). In the laboratory examination, renal dysfunction and anemia may be shown if the patient has urinary tract obstruction (7). Furthermore, it has been reported that about $60 \%$ of cases with RPF have higher levels of C-reactive protein 
and anti-nuclear antibody (3). Accordingly, primary RPF is regarded as a kind of autoimmune disease in recent years. Based on our experience, this patient might have the typical tissue structure of RPF on CT and MRI, but no urinary tract obstruction and renal dysfunction, or elevation of serum inflammatory markers. Therefore, we thought that in this patient RPF might have been due to a secondary cause.

It has been reported that about $30 \%$ of cases with RPF had a secondary cause such as drugs, malignant disease, infections, radiotherapy, and abdominal surgery $(3,5)$. According to those reports, the drugs, which are regarded as a cause of RPF, are the following: methysergide, bromocriptine, ergotamine, hydralazine, methyldopa, and betablockers. The present patient received oral administration of beta-blocker (atenolol $50 \mathrm{mg} /$ day) for treatment of hypertension for 3 years. Therefore, we discontinued atenolol, and started oral preparations of enarapril ( $5 \mathrm{mg} /$ day) for hypertension.

There are many clinical reports about the effectiveness of steroid therapy for the treatment of RPF. Ilie et al reported that oral administration of predonisolone at a dose of 40 $\mathrm{mg} /$ day for a month was responded well for the regression of proliferated retroperitoneal tissue (10). Vaglio et al also reported the treatment strategy of low dose oral preparations of predonisolone were as follows: $1 \mathrm{mg} / \mathrm{kg} /$ day for 4 to 6 weeks, and tapered gradually for a few months to $10 \mathrm{mg}$ / day, and continued a maintenance dose until 6 to 18 months (3). Furthermore, it has been reported that preparations with tamoxifen, azathioprin, cyclophosphamide, and cyclosporine are effective for the treatment of steroidresistant RPF (13-16). As with these reports, we started oral predonisolone therapy for the present case at a dose of 30 $\mathrm{mg} /$ day for 4 weeks after the diagnosis of RPF. Four weeks after the treatment with predonisolone, his proliferated retroperitoneal structure significantly regressed on follow-up CT in comparison with that on admission. Low-dose predonisolone therapy might be effective for the treatment of

\section{Japanese adult RPF.}

The effect of the urinary tract stent implantation to restrain progression of renal dysfunction has been recognized (10). On the other hand, it has not been clearly shown that whether venous stent implantation is useful to improve leg vein compression by proliferated retroperitoneal tissue or not. In the present case, right leg pain and edema of this patient worsened rapidly after the admission. It had been generally thought that at least one month is necessary to get an effect of the steroid therapy. Therefore, we had the need to remove localized venous stenosis as soon as possible. We selected a balloon-expanding type stent that is commonly used to remove severe arterial stenosis in cases with arteriosclerosis obliterans (SMART stent ${ }^{\mathrm{R}}$ ), and determined reference of the stent size to the diameter of the just proximal site of external iliac vein $(9 \mathrm{~mm})$. Percutaneous transvascular thombus capture using an inferior vena cava filter catheter is thought to be a useful procedure to prevent pulmonary embolism while performing stent implantation for iliac vein stenosis. In the present case, right external iliac vein had severe stenosis and filling delay of contrast media but not thrombus formation on venography and 3-directional CT angiography. Therefore, we did not use inferior vena cava filter catheter while performing venous stent implantation. As a result, his leg edema and pain disappeared 2 days after the stent implantation with no complications. According to our experience, percutaneous venous stent implantation might be a safe and effective procedure to improve localized leg vein stenosis in cases with RPF.

We have reported here an adult case of right external iliac vein stenosis due to RPF that was improved by treatment with percutaneous transvenous stent implantation and lowdose oral administration of predonisolone. It has been recognized that the 5 year mortality of this disease is low (about $10 \%$ ), however, the recurrence rate is relatively high (17). It might be thought that careful follow-up and more experiences is necessary to prevent the recurrent RPF.

\section{References}

1. Ormond JK. Bilateral ureteral obstruction due to envelopment and compression by an inflammatory retroperitoneal process: report of two cases. J Urol 59: 1072-1079, 1948.

2. Demko TM, Diamond JR, Groff J. Obstructive nephropathy as a result of retroperitoneal fibrosis: a review of its pathogenesis and associations. J Am Soc Nephrol 8: 684-688, 1997.

3. Vaglio A, Salvarani C, Buzio C. Retroperitoneal fibrosis. Lancet 367: 241-251, 2006.

4. van Bommel EF. Retroperitoneal fibrosis. Neth J Med 60: 231242, 2002.

5. Koep L, Zuidema GD. The clinical significance of retroperitoneal fibrosis. Surgery 81: 250-257, 1997.

6. Baker LR, Mallinson WJ, Gregory MC, et al. Idiopathic retroperitoneal fibrosis. A retrospective analysis of 60 cases. Br J Urol 60: 497-503, 1987.

7. Feinstein RS, Gatewood OM, Goldman SM, Copeland B, Walsh PC, Siegelman SS. Computerized tomography in the diagnosis of retroperitoneal fibrosis. J Urol 126: 255-259, 1981.

8. Dalla-Palma L, Rocca-Rossetti S, Pozzi-Mucelli RS, Rizzatto G.
Computed tomography in the diagnosis of retroperitoneal fibrosis. Urol Radiol 3: 77-83, 1981.

9. Arrivé L, Hricak H, Tavares NJ, Miller TR. Malignant versus nonmalignant retroperitoneal fibrosis: differentiation with MR imaging. Radiology 172: 139-143, 1989.

10. Ilie CP, Pemberton RJ, Tolley DA. Idiopathic retroperitoneal fibrosis: the case for nonsurgical treatment. BJU Int 98: 137-140, 2006.

11. Van Bommel EF, Hendriksz TR, Huiskes AW, Zeegers AG. Brief communication: tamoxifen therapy for nonmalignant retroperitoneal fibrosis. Ann Intern Med 144: 101-106, 2006.

12. Ozener C, Kiriş S, Lawrence R, Ilker Y, Akoğlu E. Potential beneficial effect of tamoxifen in retroperitoneal fibrosis. Nephrol Dial Transplant 12: 2166-2168, 1997.

13. Marcolongo R, Tavolini IM, Laveder F, et al. Immunosuppressive therapy for idiopathic retroperitoneal fibrosis: a retrospective analysis of 26 cases. Am J Med 2004 116: 194-197, 2004.

14. Marzano A, Trapani A, Leone N, Actis GC, Rizzetto M. Treatment of idiopathic retroperitoneal fibrosis using cyclosporin. Ann Rheum Dis 60: 427-428, 2001. 
15. Jois RN, Kerrigan N, Scott DG. Mycophenolate mofetil for maintenance of remission in idiopathic retroperitoneal fibrosis. Rheumatology 46: 717-718, 2007.

16. Scheel PJ Jr, Piccini J, Rahman MH, Lawler L, Jarrett T. Combined prednisone and mycophenolate mofetil treatment for retroperitoneal fibrosis. J Urol 178: 140-144, 2007.
17. Van Bommel EF, Siemes C, Hak LE, van der Veer SJ, Hendriksz TR. Long-term renal and patient outcome in idiopathic retroperitoneal fibrosis treated with prednisone. Am J Kidney Dis 49: 615$625,2007$.

(C) 2010 The Japanese Society of Internal Medicine http://www.naika.or.jp/imindex.html 